$$
\begin{gathered}
\text { NK } \\
3712 \\
\text { N5C411 } \\
1956 \\
\text { CHMP迎 }
\end{gathered}
$$



"Thirty spokes are made one by holes in a hub By vacancies joining them for a wheel's use;

The use of clay in molding pitchers

Comes from the hollow of its absence;

Doors, windows, in a house,

Are used for their emptiness:

Thus we are helped by what is not

To use what is."

$$
\text { Lao - Tse }
$$

Cover photos: Upper right: Sigrid Hahn. Lower left: Marilyn Fox. Left to right: Plate-Madeleine Vermes; Flower Pot-Bertha Mason; Vase-Gertrud Englander; Bird-Muriel Turoff; Vase-Bea Binger; Bottle-Polia Pillin; Sculpture: Skywatch-Jean Woodham. 


\section{NEW YORK SOCIETY OF CERAMIC ARTS}

$62^{\text {nd }}$ ANNUAL EXHIBITION

C E R A M I C S
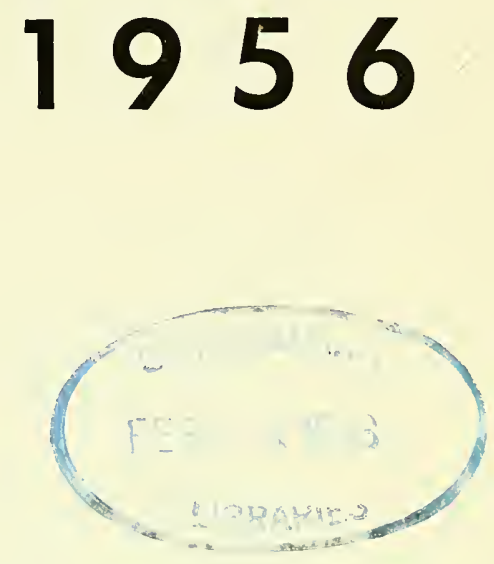

M A R C 2 nd - 2 2nd

Open to the Public:

Monday, Wednesday, Friday, Saturday 10.5

Tuesdays and Thursdays 10 AM - 10 PM

closed Sundays

Cooper Union Museum for the Arts of Decoration $4^{\text {th }}$ Avenue and $7^{\text {th }}$ Street, New York City 


\section{JURY}

Sculpture: Bea Croll, Ellen Key-Oberg, Joseph Konzal, Jane Wasey, Winifred Lansing, alternate.

Non-Member: Martin Craig. Cooper Union Art School.

Pottery: Anne de Carmel, James Crumrine, Alexandra Kasuba, Oppi Untracht, Muriel P. Turoff, alternate.

Non-Member: Calvin S. Hathaway, Director, Cooper Union Museum.

\section{Illustrated Lecture}

Thursday, March 15, 8 P.M. at the Museum

CERAMICS 1956 AND BEFORE

Hedy Backlin, Cooper Union Museum

\section{DEMONSTRATIONS}

\section{SATURDAY, MARCH 3rd}

$\begin{array}{llr}\text { 12:00 } & \text { Enameling } & \text { Gertrude Hartman } \\ \text { 2:30 P.M. } & \text { Wheel-Throwing } & \text { Jack D. Wolfe }\end{array}$

TUESDAY, MARCH 6 th

8:00 P.M. Work with Glass Maurice Heatan

\section{THURSDAY, MARCH 8th}

8:00 P.M. Wheel-Thrown Pottery

and Sculpture Rose Krebs

\section{SATURDAY, MARCH 10th}

$\begin{array}{llr}\text { 12:00 } & \begin{array}{l}\text { Various Techniques in Clay } \\ \text { 2:30 P.M. }\end{array} & \begin{array}{r}\text { Rolf Key-Oberg } \\ \text { Sculpture }\end{array} \\ \text { TUESDAY, MARCH } & \text { 13th } & \\ \text { 8:00 P.M. } & \text { Sculpture } & \text { Jane Wasey } \\ \text { SATURDAY, MARCH 17th } & \\ \text { 12:00 } & \text { Tile Decorating } & \\ \text { 2:30 P.M. } & \text { Wheel-Throwing } & \text { Marilyn Fax } \\ & \text { James Crumrine }\end{array}$

TUESDAY, MARCH 20th
8:00 P.M.
Decorating Techniques
Dido Smith

Potters Wheel: Courtesy Craftool, Inc.

Photos by Peter Adelberg 


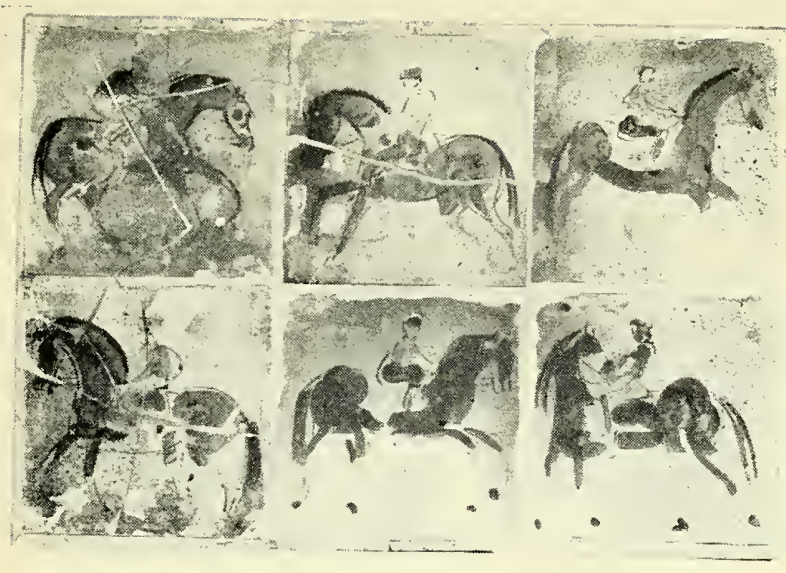

Boris Chatman: Tile Panel

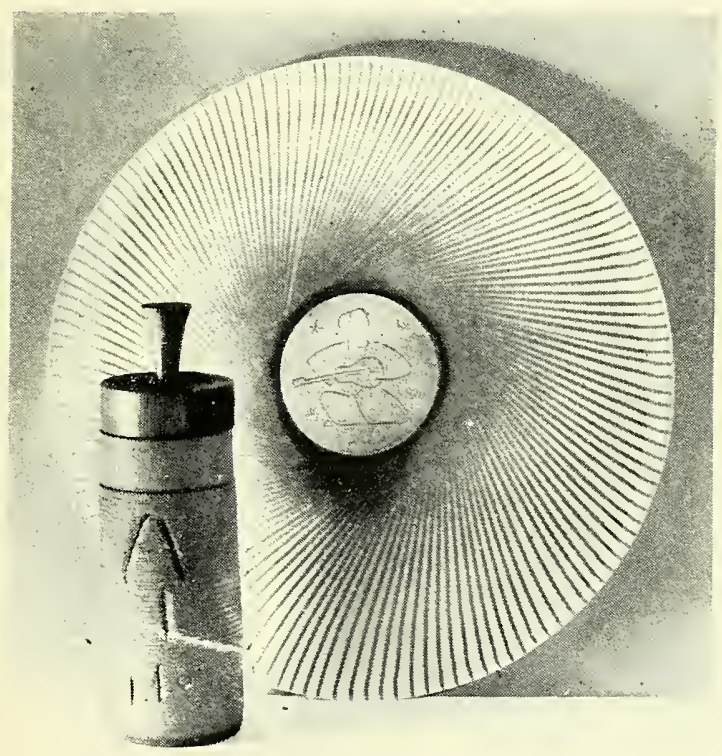

Chris Purdue:

Maurice Heaton:

Jar with Walnut Lid

Glass Cake Plate 
EXECUTIVE BOARD 1955-56

President

1 st Vice-President

2nd Vice-President

Treasurer

Corresponding Secretary

Recording Secretary

Membership Chairman

Ways \& Means Chairman

Publicity Chairmen

Special Events Chairmen

Exhibition Chairmen
Gertrude Hartman

Billy Bacharach

Dorothy Fish

Dorothy Jervis

Geraldine Driscoll

Francine Fierstein

Rozsika Blackstone

Alexandra Kasuba

Elizabeth Cowan, Oppi Untracht Roberta Leber, Gertrud Englander Rose Krebs, Freda Zuend

MEMBERSHIP
P. - Pottery
S. - Sculpture T. - Teacher
E. - Enamel
* -Exhibitor

\section{MRS. MAURICE AUSTIN}

21 Avon St., Larchmont, N. Y.

\section{BILLY BACHARACH}

1185 Park Avenue, New York City, AT 9-2328

* Geraldine BALSAM P. T.

136 Flatbush Ave., Brooklyn 17, N. Y., ST 3-5295

* B. BINGER

P. T.

58-20-207th Street, Bayside 64, L. I., N. Y., BA 9-3405

\section{* ROZSIKA B. BLACKSTONE}

412 E. 84th St., New York, N. Y., RH 4-4924

* PHYLLIS BLUNDELL P. S. T.

333 E. 49th St., New York, N. Y., PL 9-2212

* JO BOYAR

17 West 12th St., New York, N. Y., Al 5-7484

P.

* EDWARD CHANDLESS

425 Ridge Rd. Apt. IN, No. Arlington, N. J., KE 2-3397

* BORIS CHATMAN

321 W. 56th St., New York 19, N. Y., CO 5-0693

MRS. THOMAS CLARKE, Jr.

620 Park Avenue, New York 21, N. Y., TR 9-4189

* BRURIA S. COLTER

749 East 175th St., Bronx 57, N. Y., TR 2-8315 
35 W. 9th St., New York 11, N. Y., GR 5-3865

* BEA CROLL

S.

11 Waverly PI., New York 3, N. Y., GR 7-9060

\section{ERICA CROMPTON}

199 E. 76th St., New York 21, N. Y.

ROBERT CRONBACH

S.

170 Henry St., Westbury, N. Y.

326 E. 49th St., New York 17, N. Y., PL 5-7448

MARY BARRETT CUMMINGS P. E. T.

33 Morton St., New York 14, N. Y., WA 4-0472

WILLIAM P. DALEY

R.F.D. \#1, New Paltz, N. Y., New Paltz 8312

WILLIAM B. DALTON

298 Ocean Drive East, Stamford, Conn., DA 3-6458

\section{SHIRLEY DE BOLES}

655 E. 14th St., New York 9, N. Y.

* ANNE DE CARMEL

P.

51 E. 65th St., New York, N. Y., RH 4-5370

PATRICIA N. DEGENER

P. T.

5600 Country Day Lane, St. Louis 21, Mo.

* Geraldine G. DRISCOll

\$.

239 E. 19th St., New York, N. Y., GR 3-1158

CHERYL ELLSWORTH

P.

P.

10 Mitchell PI., New York 17, N. Y., PL 5-1628

* GERTRUD ENGLANDER

P. T.

84-51 Beverly Rd., Kew Gardens 15, N. Y., VI 7-5125

CARL ESPENSCHEID

R.F.D. 2, Lebanon, N. J.

P.

FRANCINE FIERSTEIN

12 Secor Road, Scarsdale, N. Y., SC 3-5390

DOROTHY FISH

P. T.

8 Huguenot Dr., Larchmont, N. Y., TE 4-2089

JACQUELINE FONG

237 E. 20th St., New York 3, N. Y., SP 7-8434

* MARILYN FOX

P. T.

1229 Cafrey Ave., Far Rockaway, N. Y., FA 7-6931

* ChARLOTTE FREEMAN

P.

P. 601 E. 20th St., Apt. 5D, New York 10, N. Y., OR 7-2784 


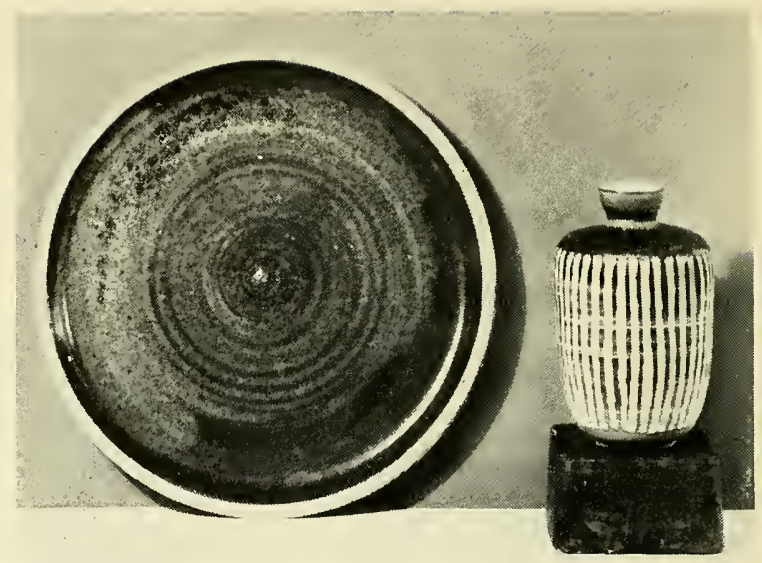

Priscilla Porter:

Dorothy Larson:

Stoneware Platter

Incised Jug
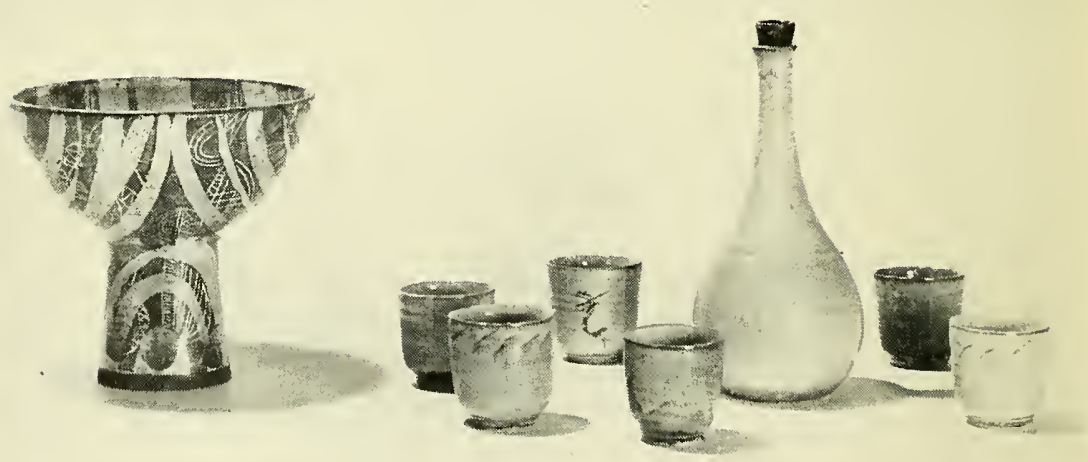

Edward Chandless: Compote

Doris de Vries Klein: Cordial Set 


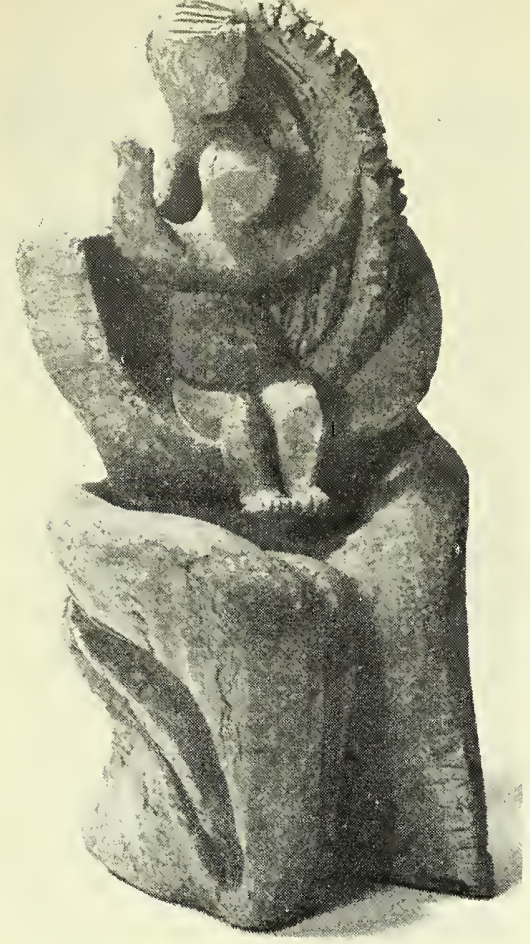

Joseph Konzal: Mother and Child

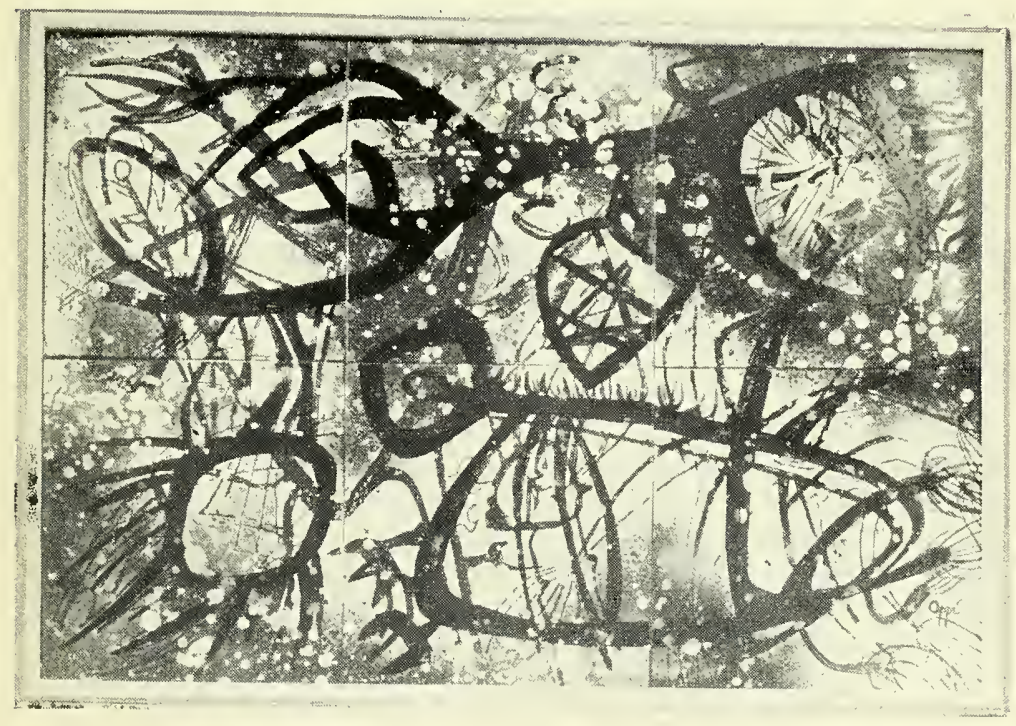

Oppi Untratht: Enamel on Steel 
138-07-90th Ave., Jamaica, N. Y.

385 Heather Lane, Hewlett Harbor, N. Y., Franklin 4-4351

\section{MANNAH GOTTESMAN}

349 Hamilton Ave., Hewlett, L. I., N. Y., Franklin 4-4164

* JOSEPH P. GREBANIER

I866 E. 4th St., Brooklyn 23, N. Y., DE 9-2709

* ESTHER GREENLEAF

27 Woodcrest Ave., Short Hills, N. J., DR 6-2556

* ALICE H. GUNDELFINGER

444 Central Park West, New York 25, N. Y., AC 2-3131

* SIGRID HAHN

796 Middleneck Rd., Great Neck, L. I., N. Y., GR 2-3569

* ESTELLE HALPER

96 Park Drive, Tuckahoe 7, N. Y., WO 1-3579

* GERTRUDE MARTMAN

P. T.

E.

115 E. 89th St., New York 28, N. Y., TR 6-9728

* MAURICE HEATON

G.

West Nyack, N. Y., Congers 8-2151

*

VIVIKA and OTTO HEINO

3204 South Hoover St., Los Angeles 7, Calif.

* NORA HERZ

P. S. T.

425 Ridge Road, No. Arlington, N. J., Kearney 2-2622

* LILI R. HIRSCH

314 East 6th St., New York 3, N. Y., AL 4-0749

HILDA HORTENS

16 Briar Hill Drive, Yonkers, N. Y., YO 5-9070

* KA KWONG HUI

P. S.

57 Underhill Ave., Brooklyn 38, N. Y., ST 9-7834

* MARGARET ISRAEL

444 West 22nd St., New York, N. Y., CH 3-6530

ALBERT D. JACOBSON P. S. T.

Seir Hill Rd., Wilton, Conn., Wilton 7-7065

* DOROTHY B. JERVIS

1155 Park Ave., New York 28, N. Y., SA 2-5467

ILSE R. JOHNSON

P. T.

Box 354, Montrose, N. Y., Peekskill 7-2821

EDNA YADVEN KAMLET

P.

P. T.

170 Second Ave., New York 2, N. Y., GR 7-4173 
104-06-217th St., Queens Village 29, N. Y., HO 4-2529

MRS. LEWIS B. KAUFMAN P. T.

43 Lynton PI., White Plains, N. Y.

* MARGOT KEMPE

P. S. T.

130 Morningside Dr., New York 27, N. Y., MO 2-0173

* ELLEN KEY-OBERG

S. T.

59 E. 3rd St., New York 3, N. Y., GR 7-7236

* ROLF KEY-OBERG

59 E. 3rd St., New York 3, N. Y., GR 7-7236

* DORIS DE VRIES KLEIN

P. T.

168 Waverly Rd., Scarsdale, N. Y., NE 2-7412

* JOSEPH KONZAL

S. T.

161 W. 23rd St., New York, N. Y., CH 2-2341

\section{ALMA KRAUS}

Stony Lodge, Ossining, N. Y.

* ROSE KREBS

P. S.

321 W. 56th St., New York 19, N. Y., CO 5-0693

WALTER D. and MARY C. KRING

P.

153 East 78th St., New York, N. Y., LY 6-5949

HELEN E. KRUGER

80 Aspen St., Floral Park, N. Y.

* WINIFRED LANSING

370 Central Park West, New York, N. Y., RI 9-6957

* DOROTHY A. LARSON

RD \#2, Washington, N. J., MU 9-0251 W 2

* ROBERTA LEBER

P. T.

- Wingarth Studios, Leber Rd., Blauvelt, N. Y. Piermont 2-2181

\section{ROBERT LEVINE}

1115 Jerome Ave., New York 52, N. Y., TE 8-7743

* PETER LIPMAN-WULF

P. S. T.

766 Sixth Avenue, New York, N. Y., CH 3-5444

* CHARLOTTE MALTEN

P. T.

74 West 68th St., New York, N. Y., SC 4-7819

* BERTHA MASON

11 Hillcrest Ave., Larchmont, N. Y., TE 4-2481

MAUD MOSS MASON

P.

National Arts Club, 15 Gramercy Park, New York, N. Y.

* LOUIS E. MENDEZ

P. S.

RD \#1, Trenton, N. J., Pennington 7-0638-J 


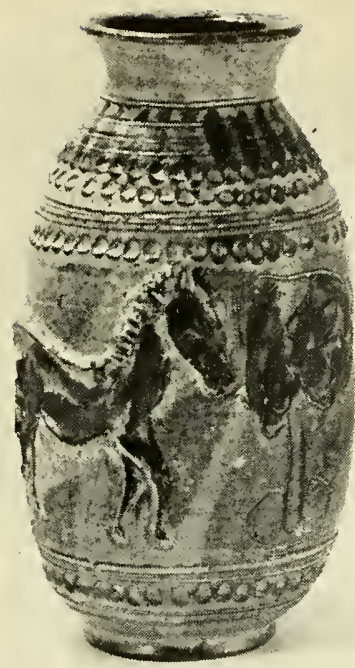

Rose Krebs: Decorated Vase

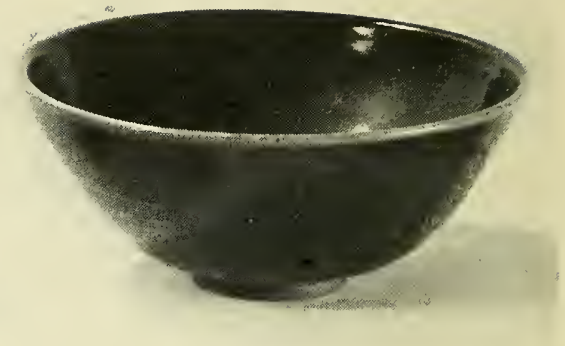

Walter and Mary Kring: Ox-blood Bowl

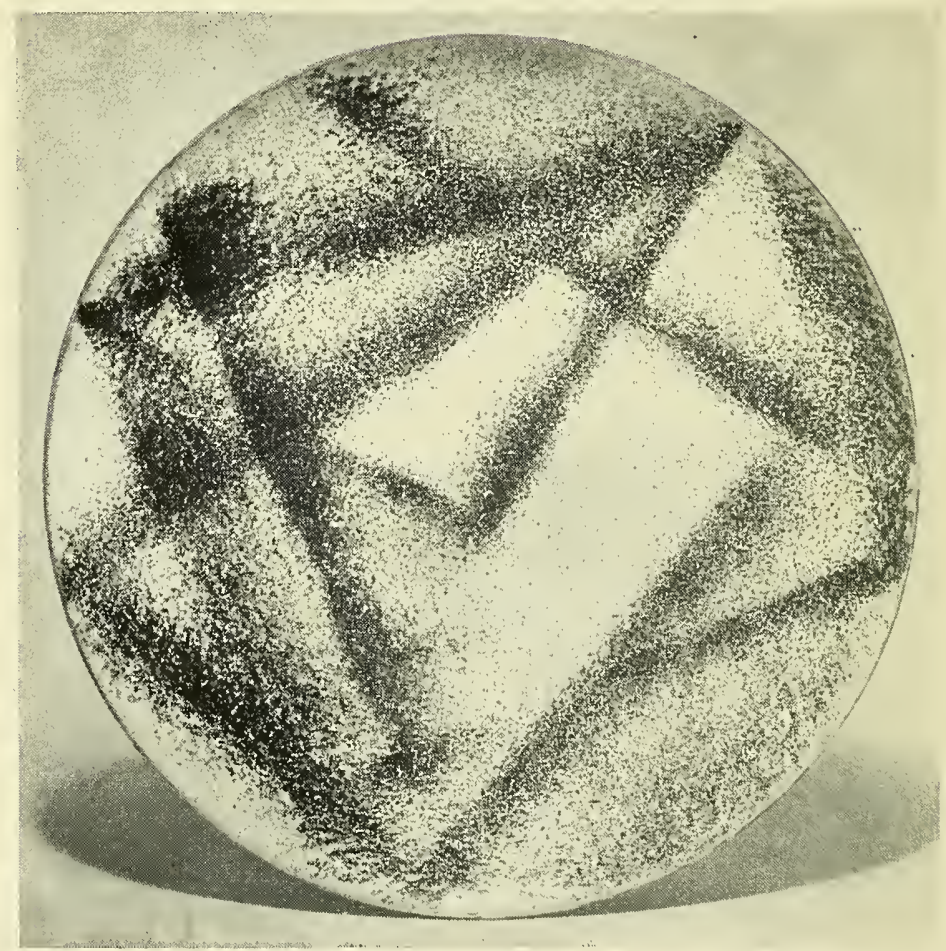




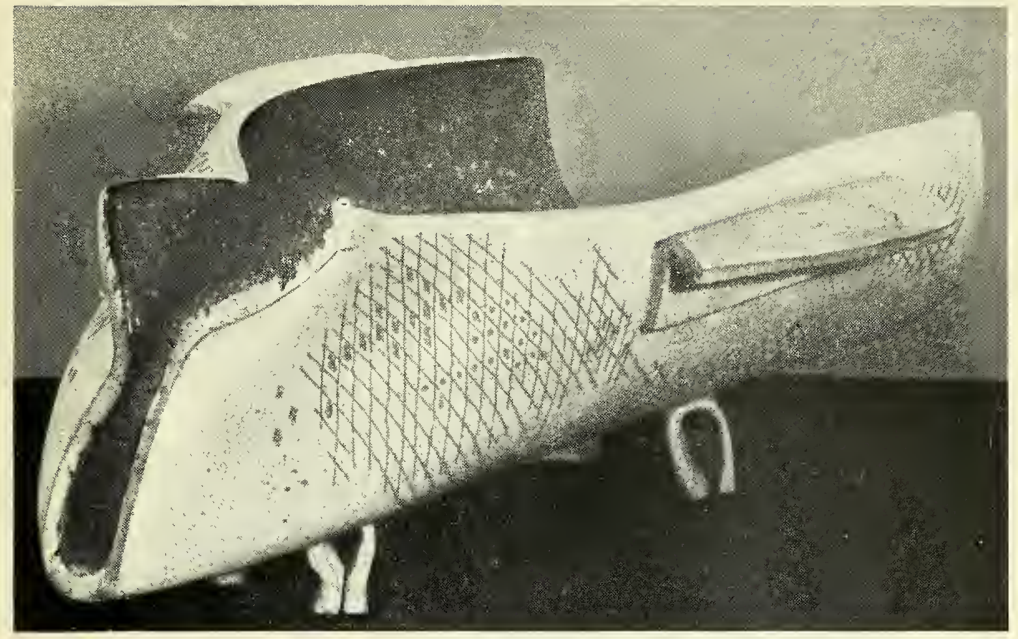

Marianna von Allesch: Armadillo
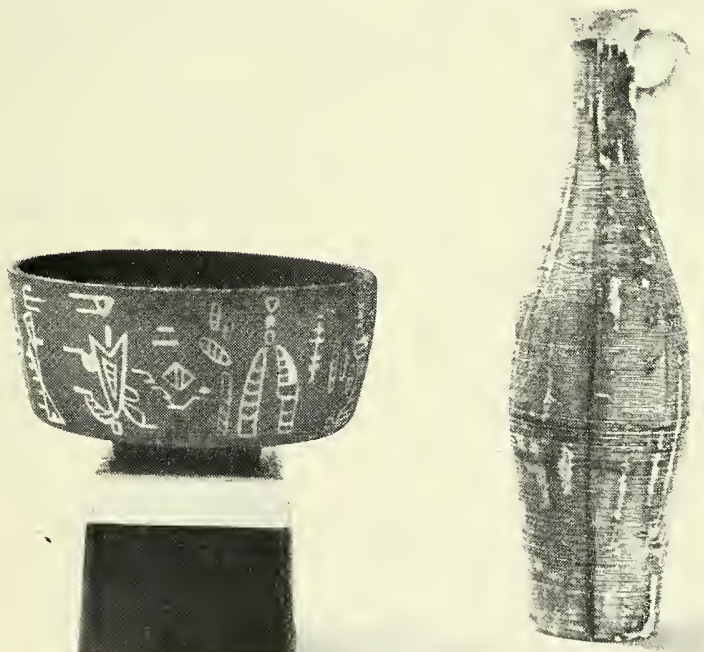

Rozsika Blacksione:

Charlotte Malten: Pitcher Sgrafitto Bowl 
237 West 1 ith St., New York 14, N. Y., CH 2-0292

HERTA MOSELSIO

P. S. T.

Old Bennington, Vermont

MARILYN NEWMARK

274 Bedell Terrace, W. Hempstead, N. Y.

HILDA NIEDELMAN

S.

875 Fifth Avenue, New York 27, N. Y.

FRITZE PASSWEG

5.

300 Central Park W., New York 24, N. Y., EN 2-0801

IVY PELISH

5.

326 Troy Avenue, Brooklyn 13, N. Y.

HELEN CLARK PHILLIPS

S.

62 Bank St., New York 14, N. Y., WA 9-2548

* POLIA PILLIN

c/O The Willow, 182 W. 4th St., New York WA 4-9220

* PRISCILla M. PORTER

P. S. T.

438 E. 57th St., New Yo:k 22, N. Y., PL 3-3872

* CHRIS PURDUE

P. 5 .

Deer Run Rd., Woodbridge 15, New Haven, Conn.

T. A. RANDALL

P. S. T.

Alfred, New York

CHARLOTTE REGESTER

P. S. T.

3 Mill Lane, Rockport, Mass.

* ARPAD ROSTI

E.

Brewster, N. Y., BR 9-4353

GRETE SCHULLER

S.

116 E. 83rd St., New York 28, N. Y., RE 4-3449

ALFARETTA D. SHIRLEY

P. T.

Box 431, Short Hills, N. J., DR 9-5462

* DIDO SMITH

P. S. T.

371 Hamilton Ave., Hewlett, N. Y., Franklin 4-1538

ELLA SPITZ

S.

1150 Fifth Ave., New York 28, N. Y., ATwater 9-4031

MRS. HUGO STIX

132 MacDougal St., New York City, GR 7-2612

BEATRICE STONE

P. 630 Park Ave., New York, N. Y.

* JULIUS TAYLOR

29 West 64th St., New York 23, N. Y., TR 7-1406

S.

P. 
721 Marshall PI., Plainfield, N. J., PLainfield 5-3613

CAROLE TERWILLIGER

P. 430 East 57th St., New York, N. Y.

N. TSCHACBASOV

P. T.

222 West 23rd St., New York, N. Y.

* MURIEL P. TUROFF

P. S. T. 5450 Netherland Ave., Bronx 71, N. Y., KI 9-7086

604 Logan St., Brooklyn 8, N. Y., AP 7-4969

109-19-72nd Ave., Forest Hills 75, N. Y., LI 4-9558

\section{LEON VOLKMAR}

433 Poplar St., Laguna Beach, Calif.

* RUTH VOLLMER

25 Central Park West, New York 23, N. Y., CO 5-2811

* MARIANNA VON ALLESCH

P. S.

50 West 13th St., New York 11, N. Y., OR 5-i885

* MARJORIE WALZER

P.

Dogwood Lane, Westport, Conn., CA 7-2093

\section{JANE WASEY}

S.

178 East 75th St., New York 21, N. Y.

\section{ERNA WEILL}

886 Alpine Dr., Teaneck, N. J., TEaneck 7-1627

ANITA WESCHLER

136 Waverly PI., New York 14, N. Y., WA 9-6378

* FRANS WILDENHAIN

R.D. Bushnells Basin, Pittsford, N. Y.

* ELLA WINTERROLL

S.P. 35-09-169th St., Flushing, N. Y., FL 3-4975

62 Horatio St., New York 14, N. Y., WA 4-6019

* JEAN WOODHAM

Wayfaring Road, Norwalk, Conn., VI 7-4246

451 Ninth St., Brooklyn 15, N. Y., HY 9-0840

FREDA ZUEND

329 E. 58th St., New York 22, N. Y., PL 8-2735 


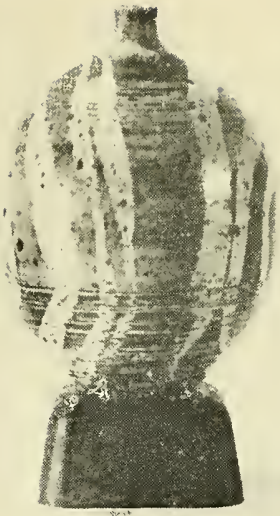

Vivika and Otto Heino: Reduction Vase

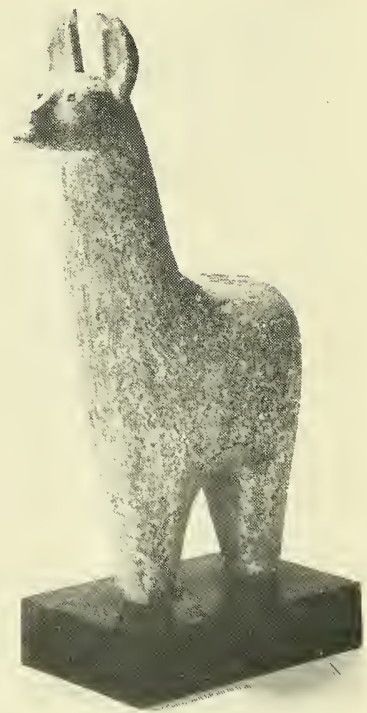

Margol Kempe: Llama

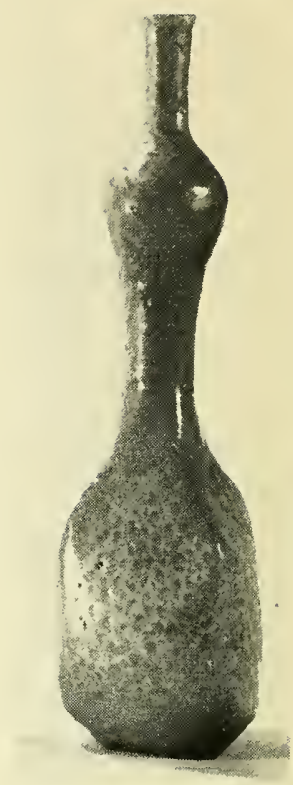

Ka Kwong Hui : Spotted Vase 


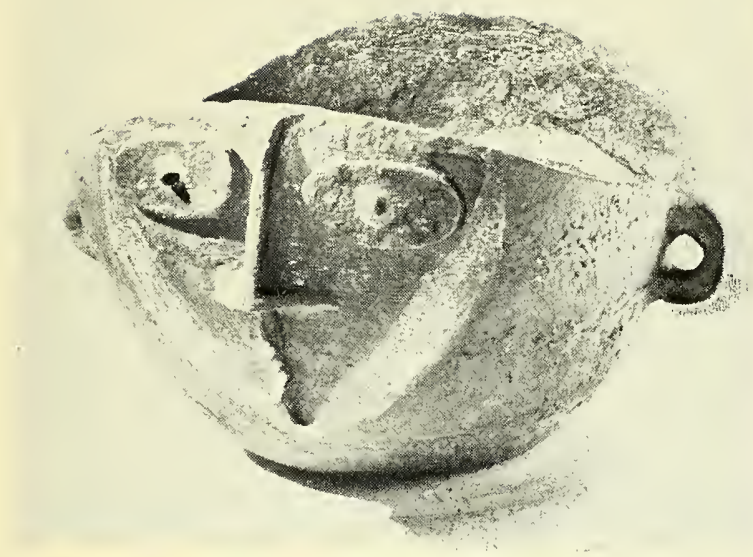

Peter Lipman-Wulf: Large Bowl

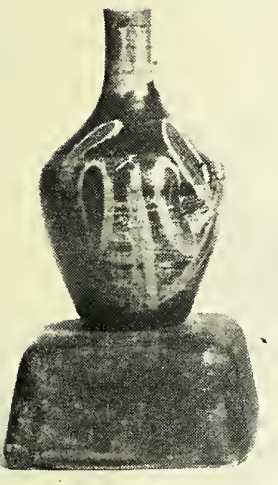

Alice Gundelfinger: Bottle with wax resist decor
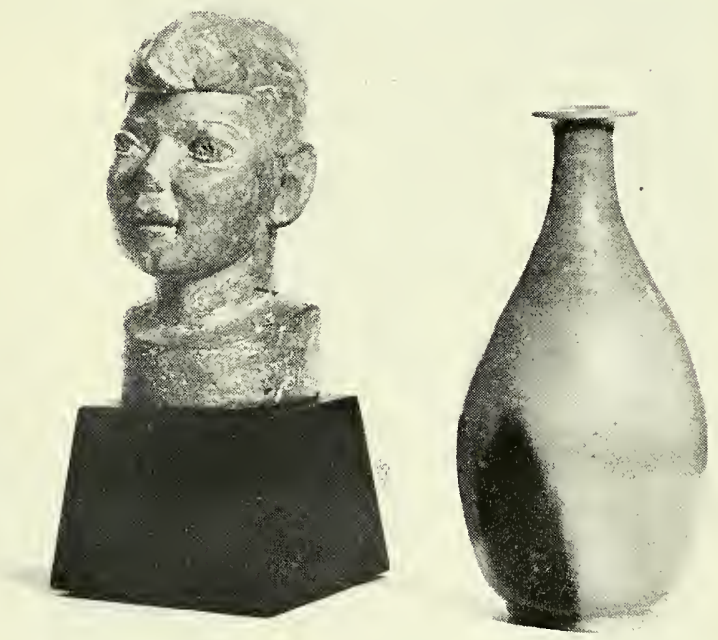

Bea Croll: Jeffry

Joseph Grebanier: Stoneware Bottle with red Patch 


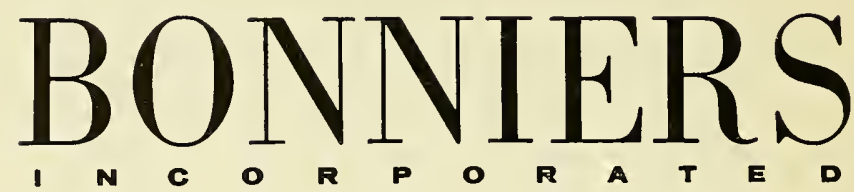

605 MADISON AVE., NEW YORK 22

\section{CRAFT STUDENTS} League of the YWCA-24rd Year

$\begin{array}{lll}\text { Painting } & \text { Bookbinding } & \text { Enameling } \\ \text { Sculpture } & \text { Silversmithing } & \text { Jewelry } \\ \text { Ceramics } & \text { Metalwork } & \text { Weaving }\end{array}$
Ceramics Metalwork Weaving Silk Screen Cabinetmaking Mẹn, Women, Day, Eve. Catalog

140 West 22nd St., N. Y. C. CH 3-5747

\section{GREENWICH HOUSE POTTERY}

16 Jones Street N.Y. 14

- Pottery - Glaze Chemistry

- Ceramic Design - Ceramic

CH 2-4106 Director Jane Hartsook

\section{MARGOT KEMPE}

130 MORNINGSIDE DRIVE, NEW YORK SCULPTOR AND INSTRUCTOR CERAMICS - METAL - JEWELRY

\section{CERAMIC AGE}

114 EAST 32nd STREET NEW YORK 13 The latest News and Articles by Outstanding Artists

Published monthly

$\$ 3.50$ for one year $\$ 6.00$ for two years
B. F. DRAKENFELD \& CO., Inc. 45 PARK PLACE, NEW YORK 10, N. Y. KILNS - CLAYS - GLAZES FOR POTTERY - GLASS - CHINA 


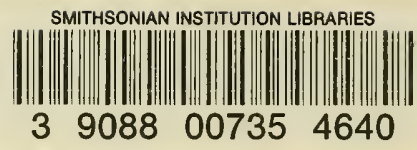

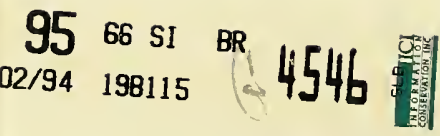





\section{$\mathrm{Cu}^{2} \mathrm{~s}$}

IIYYYQ17

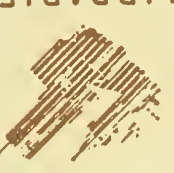

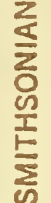
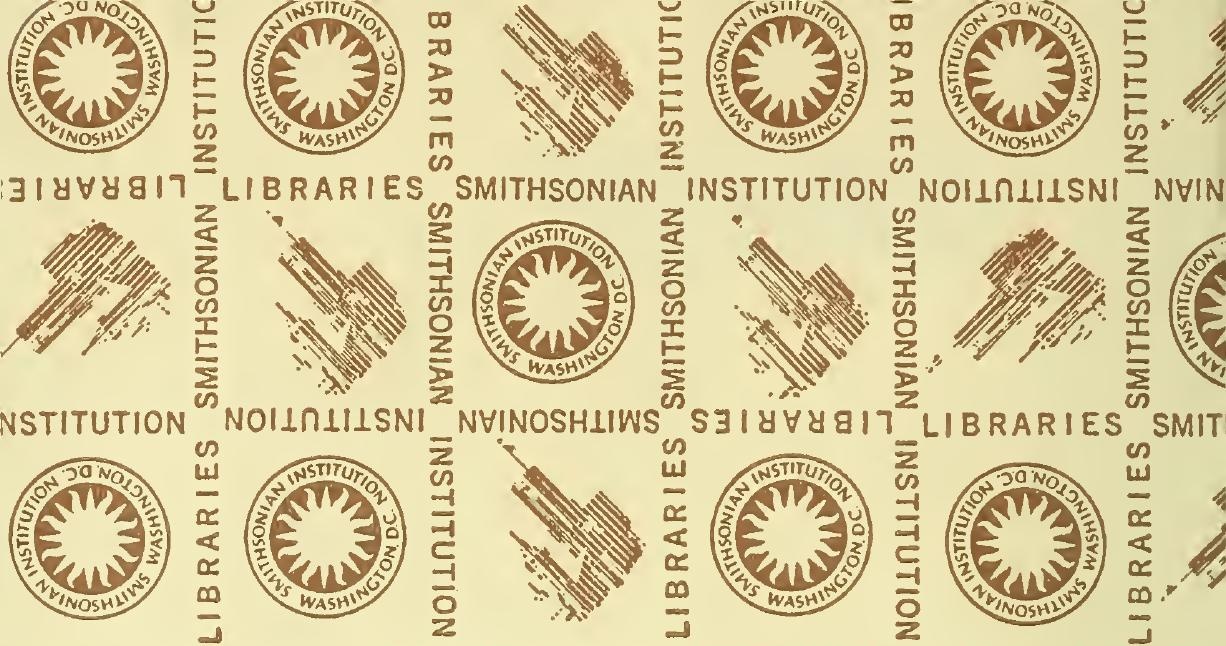

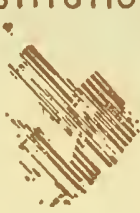

S
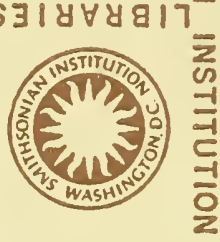

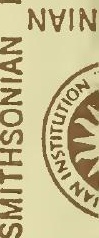

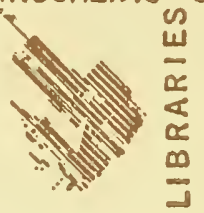
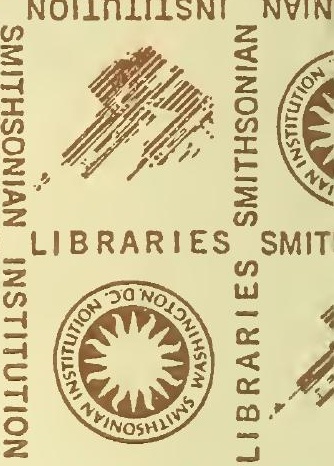
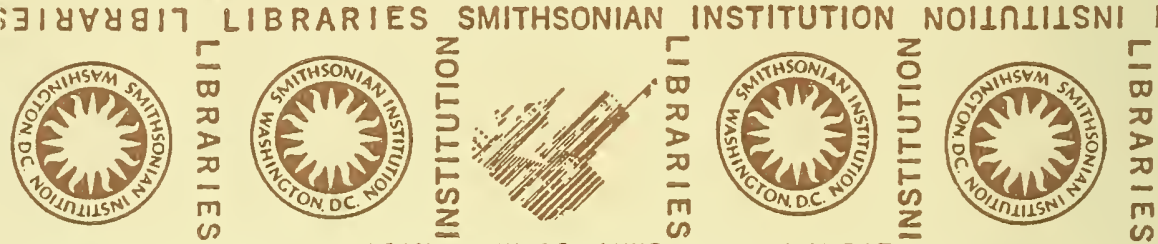

NOIIRIIISNI NHINOSHIIWS S3IVHY817 LIBRARIES SMIT
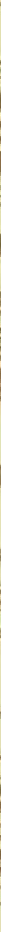

NSTITUTION

NOIINLILSN

NVINOSHIIWS

s:

$\overline{2}$
$\stackrel{3}{\exists}$
$=$ SIIYVY817 LIBRARIES SMIT

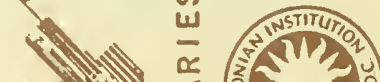

$\sum_{n}$

\% 

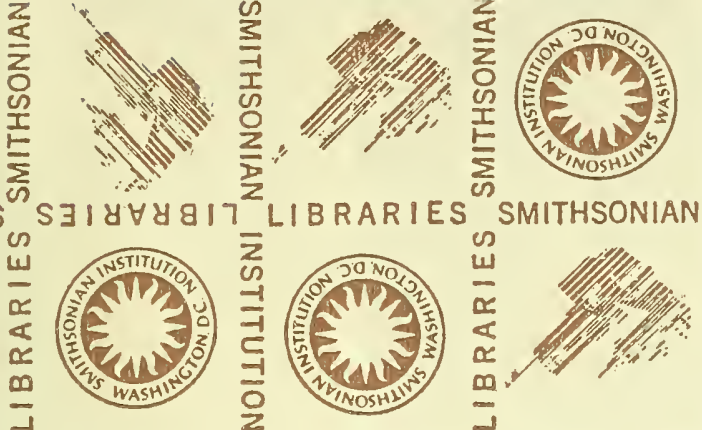

LIBRARIES

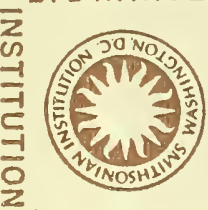

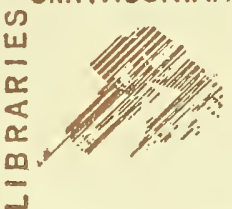

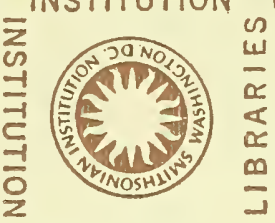

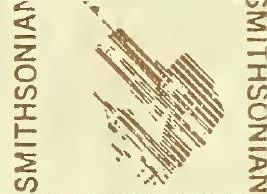

NOIINLILSNI

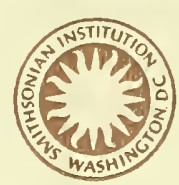

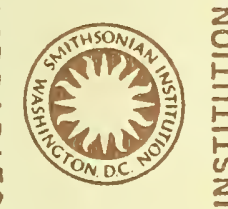
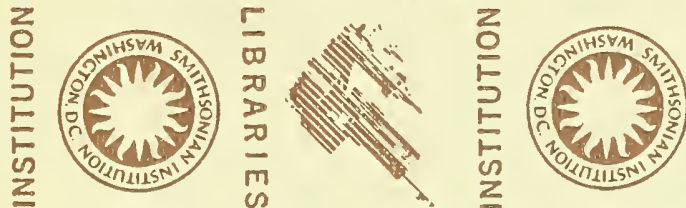

$\infty$
$\infty$
$\infty$
$\infty$
$m$
0

LIBRARIES

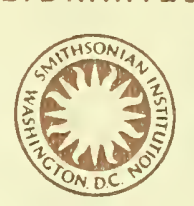

NOILOLILSNI
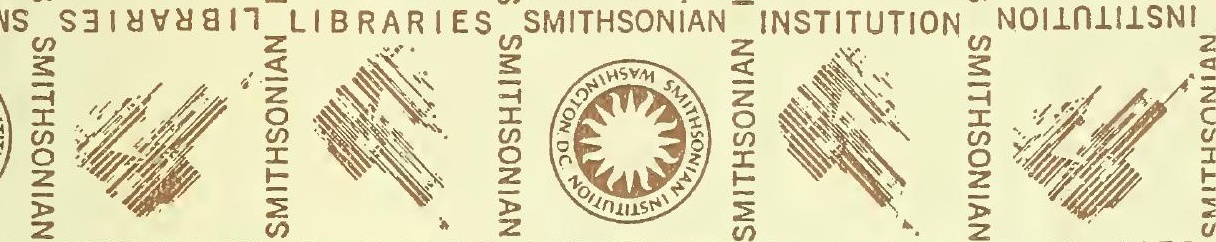

$A N$
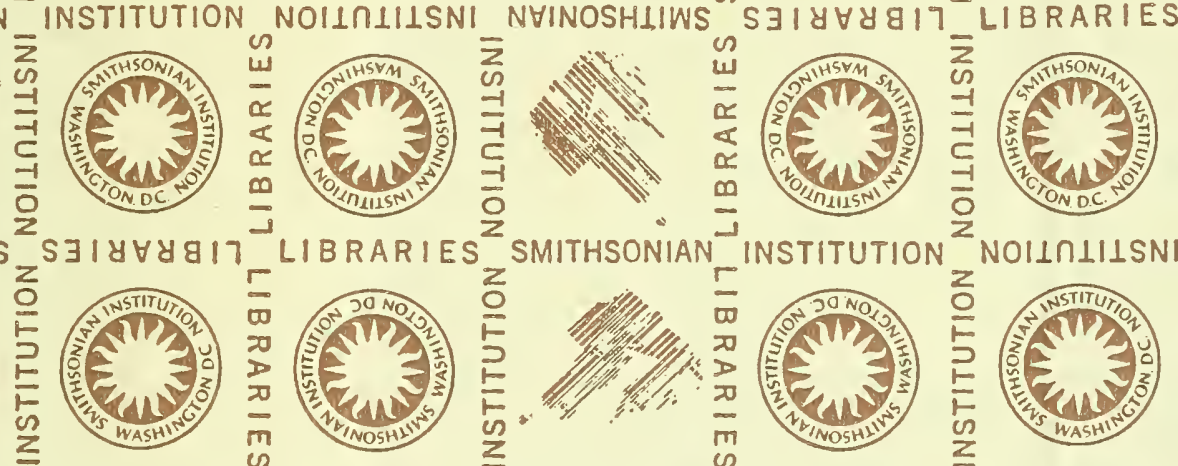

NOIINIIISNI
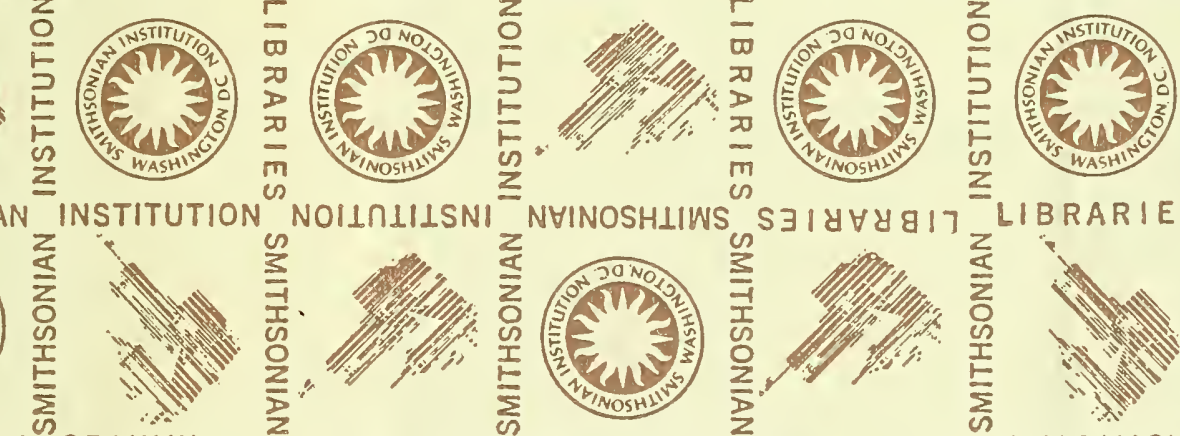

LIBRARIES

WS S $I$ I Y 84817 LIBRARIES SMITHSONIAN
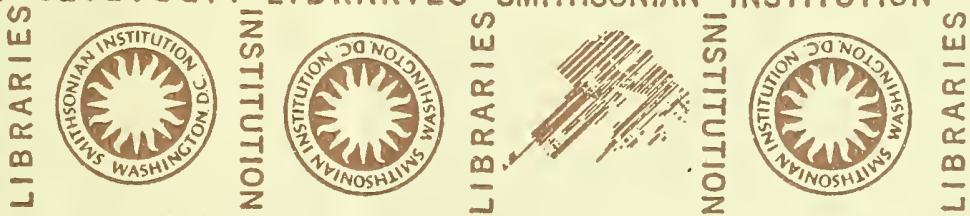

NOILIIIISNI

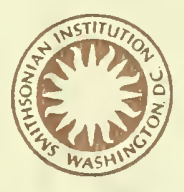

LIBRARIES 
4.

(4) 16:

W.1 2 - 